\title{
ICD-9 code reporting among patients from the Minnesota SARI surveillance program
}

\author{
Andrea Steffens ${ }^{* 1}$, Hannah Friedlander ${ }^{2}$, Kathy Como-Sabetti ${ }^{2}$, Dave Boxrud ${ }^{2}$, \\ Sarah Bistodeau², Anna Strain ${ }^{2}$, Carrie Reed ${ }^{1}$, Ruth Lynfield ${ }^{2}$ and Ashley Fowlkes ${ }^{1}$
}

${ }^{1}$ Influenza Division, Centers for Disease Control and Prevention, Atlanta, GA, USA; ${ }^{2}$ Minnesota Department of Health, Saint Paul, MN, USA

\section{Introduction}

The ICD-9 codes for acute respiratory illness (ARI) and pneumonia/influenza (P\&I) are commonly used in ARI surveillance; however, few studies evaluate the accuracy of these codes or the importance of ICD-9 position. We reviewed ICD-9 codes reported among patients identified through severe acute respiratory infection (SARI) surveillance to compare medical record documentation with medical coding and evaluated ICD-9 codes assigned to patients with influenza detections.

\section{Methods}

The Minnesota Department of Health (MDH) conducted SARI surveillance at three hospitals. All hospitalized patients with submission of a physician-ordered upper respiratory specimens (e.g., sputum, throat or nasopharyngeal swabs) were enrolled. A medical chart review was conducted to identify those meeting SARI criteria, defined as patients admitted to an inpatient ward with new onset of respiratory symptoms or acute exacerbation of chronic respiratory conditions. Enrolled patients who did not meet the SARI criteria were categorized as non-SARI. Residual material from the upper respiratory specimens were submitted to MDH for influenza testing by RT-PCR. Demographic and clinical data, including up to eight ICD-9 codes, were collected through the medical record review. Patients with an ICD-9 code indicating ARI (460 to 466) or P\&I (480 to 488) were defined as having an ARI/P\&I code. We compared the frequency of ARI/P\&I codes by SARI clinical criteria and influenza detection and evaluated the position of the reported ARI/P\&I code.

\section{Results}

From May 2013 through August 2015, we enrolled 5,950 patients, of which 4,449 (75\%) met SARI criteria and 1501 did not (non-SARI). An ARI/P\&I code in any position was found in $61 \%$ (2705) of SARI vs. $16 \%$ (241) of non-SARI patients (odds ratio [OR] 8.1, 95\% confidence interval [CI] 7.0-9.4); an ARI/P\&I code in the first position was found in $40 \%$ of SARI vs $7 \%$ of non-SARI patients $(\mathrm{OR}=8.6,95 \%$ CI 7.0-10.5). Among SARI patients with at least one ARI/P\&I code, $66 \%$ had their first or only ARI/P\&I code in the $1^{\text {st }}$ position, $25 \%$ in the $2^{\text {nd }}$ position, and $6 \%$ in the $3^{\text {rd }}$ position. For identification of SARI, sensitivity/specificity was $61 \% / 84 \%$ for ARI/ P\&I codes in any position and $40 \% / 93 \%$ for ARI/P\&I codes in the $1^{\text {st }}$ position. Among SARI patients, codes for pneumonia (486) and acute bronchiolitis $(466.11,466.19)$ were commonly reported. The most frequent codes among SARI patients without an ARI/P\&I code were fever (780.6), acute respiratory failure (518.81), and asthma (493.92) (Table). Influenza was detected among $8 \%$ (351) of SARI patients. An ARI/P\&I code in any position was more common in influenzapositive vs. influenza-negative SARI patients ( $77 \%$ vs 59\%, OR 2.4 , $95 \%$ CI 1.8-3.1). An ARI/P\&I code in the $1^{\text {st }}$ position was slightly more common in influenza-positive vs -negative patients though not significant (44\% vs $40 \%)$.

\section{Conclusions}

Among patients from whom a respiratory specimen was collected, administrative data identified those meeting SARI with moderate sensitivity and high specificity, and with lower sensitivity but greater specificity when limited to the $1^{\text {st }}$ ICD-9 position. Pneumonia and acute bronchiolitis ICD-9 codes were frequent ARI/P\&I codes among SARI patients. Further investigation is needed to determine the value of including additional ICD-9 codes, such as respiratory distress and acute asthma exacerbation, in identifying SARI.

Most frequently reported ICD-9 codes among SARI, non-SARI and influenza test-positive patients with no ARI/P\&I codes.

\begin{tabular}{|c|c|c|c|}
\hline Patient Group & ICD-9 Code & Description & Frequency N(\%) \\
\hline \multirow{5}{*}{ SARI by Chart Review } & 780.60 & Fever, unspecified & $247(14)$ \\
\cline { 2 - 4 } & 518.81 & Acute respiratory failure & $188(11)$ \\
\cline { 2 - 4 } & 786.09 & Other respiratory abnormalities & $143(8)$ \\
\cline { 2 - 4 } & 493.92 & Asthma, unspecified type, with (acute) exacerbation & $124(7)$ \\
\cline { 2 - 4 } Non-SARI by Chart Review & 518.82 & Other pulmonary insufficiency, not elscwhere classified & $110(6)$ \\
\hline & 780.60 & Fever, unspecificd & $205(16)$ \\
\hline & 780.39 & Other convulsions & $62(5)$ \\
\hline & 518.81 & Acute respiratory failure & $61(5)$ \\
\hline & 276.51 & Dehydration & $57(5)$ \\
\hline \multirow{5}{*}{ Influenza-Positive } & 599.0 & Urinary tract infection, site not specified & $50(4)$ \\
\hline & 518.81 & Acute respiratory failure & $15(13)$ \\
\hline & 780.60 & Fever, unspecified & $13(11)$ \\
\cline { 2 - 4 } & 401.9 & Unspecified essential hypertension & $9(8)$ \\
\hline & 428.0 & Congestive heart failure & $8(7)$ \\
\hline & 038.9 & Unspecified septicemia & $7(6)$ \\
\hline
\end{tabular}

Keywords

ICD-9; Influenza; Hospitalized; Respiratory

\section{*Andrea Steffens}

E-mail: vqg1@cdc.gov 